\title{
Nonrelativistic Solutions of Morse Potential from Relativistic Klein-Gordon Equation
}

\author{
Hosung Sun \\ Department of Chemistry, Sungkyunkwan University, Suwon 440-746, Korea.E-mail: hsun@skku.edu \\ Received August 12, 2010, Accepted September 27, 2010
}

\begin{abstract}
Recently it is suggested that it may be possible to obtain the approximate or exact bound state solutions of nonrelativistic Schrödinger equation from relativistic Klein-Gordon equation, which seems to be counter-intuitive. But the suggestion is further elaborated to propose a more detailed method for obtaining nonrelativistic solutions from relativistic solutions. We demonstrate the feasibility of the proposed method with the Morse potential as an example. This work shows that exact relativistic solutions can be a good starting point for obtaining nonrelativistic solutions even though a rigorous algebraic method is not found yet.
\end{abstract}

Key Words: Relativistic Klein-Gordon equation, Morse potential

\section{Introduction}

For a spinless particle of rest mass $m$, the relativistic onedimensional time-independent Klein-Gordon equation is ${ }^{1-3}$

$$
-\hbar^{2} c^{2} \frac{d^{2}}{d x^{2}} \Psi(x)+\left[m c^{2}+S(x)\right]^{2} \Psi(x)=[E-V(x)]^{2} \Psi(x)
$$

where $E$ is the total energy of the particle, $c$ is the velocity of light, and $\hbar$ is the Planck constant divided by $2 \pi . V(x)$ is called the Lorentz vector potential that is the time-component of the $(1+1)$-vector potential $[A(t, x)]$ when the space component of the vector potential is chosen to vanish. $S(x)$ is called the Lorentz scalar potential that couples to the space-time scalar potential $[S(t, x)]$. In order to understand the relativistic effects in nuclear chemistry or physics, the bound state solutions of the Klein-Gordon equation have been frequently investigated. For the potentials such as linear, ${ }^{4-6}$ exponential, ${ }^{5-8}$ Coulomb, ${ }^{9,10} \mathrm{Hul}-$ thén, ${ }^{11-14}$ Rosen-Morse, ${ }^{15,16}$ etc., the exact bound state solutions of the one-dimensional Klein-Gordon equation have been reported. It is also reported that the one-dimensional Klein-Gordon equation with shape invariant vector and scalar potentials can be exactly solved. ${ }^{3-7,11,17}$ The approximate bound state solutions of the Klein-Gordon equation for more complex potentials or for one-dimensional potentials with centrifugal term are also investigated. ${ }^{11,18-23}$

The nonrelativistic Schrödinger equation corresponding to the above relativistic Klein-Gordon equation is

$$
-\frac{\hbar^{2}}{2 m} \frac{d^{2}}{d x^{2}} \Psi^{\text {nonrel }}(x)+W(x) \Psi^{\text {nonrel }}(x)=E^{\text {nonrel }} \Psi^{\text {nonrel }}(x)
$$

where the potential $W(x) \equiv S(x)+V(x)$. In the relativistic Klein-Gordon equation, the vector potential $V(x)$ couples to the energy while the scalar potential $S(x)$ couples to the mass of the particle. The two couplings are independent and intrinsically different. But, in the nonrelativistic Schrödinger equation, the vector and scalar potentials are treated on a same footing, i.e., they are no more distinguishable.

As seen in Eq. (2), the nonrelativistic Schrödinger equation is bosonic in nature, i.e., spin does not involve in it. The relativistic Klein-Gordon equation is also for a spinless particle. It implicitly suggests that there may be a certain relationship between the solutions of the two fundamental equations. For some potential the exact relativistic solutions can be obtained from the Klein-Gordon equation but the corresponding nonrelativistic Schrödinger equation is not necessarily solvable for the same potential. Therefore, the relationship (if one ever finds it) will be usefully utilized to obtain the solutions of the insolvable Schrödinger equation. Chen et al. ${ }^{10}$ also noted that there is a possibility of obtaining approximate nonrelativistic solutions from relativistic ones but it requires more profound investigation. Though the possibility of finding the bound state solutions of nonrelativistic Schrödinger equation from the relativistic Klein-Gordon equation has been sought after for some time, any definite and systematic method has not been found yet.

However, very recently we proposed a little bit crude but meaningful approach for deriving the bound state solutions of nonrelativistic Schrödinger equation from the bound state solutions of relativistic Klein-Gordon equation. ${ }^{25}$ The essence of the approach was that, in the nonrelativistic limit, the Schrödinger equation may be derived from the Klein-Gordon equation when the two potential energies $((|S|$ and $(|V|))$ are small compared to the rest mass energy $m c^{2}$, then the nonrelativistic energy is approximated to $E^{\text {nonrel }} \cong E-m c^{2}$ and the nonrelativistic eigenfunction is that $\Psi^{\text {nonrel }}(x) \cong \Psi(x)$. That is, the nonrelativistic eigenenergies $E^{\text {nonrel }}$ can be determined by taking the nonrelativistic limit values of the relativistic eigenenergies $E$. We, in the former work, applied the simple idea to the harmonic oscillator and the Coulomb potentials and successfully demonstrated the feasibility of the approach.

In this work, we elaborate our former approach and suggest a step-by-step method in detail. To show the possibility of the suggested method, it is applied to the Morse potential. Since the exact bound state solutions of the nonrelativistic Schrödinger equation for the Morse potential are known, the Morse potential is an appropriate example to analyze the nonrelativistic solutions 
obtained from the relativistic Klein-Gordon equation without ambiguity. During the course of the derivation, the relativistic effect (amount of relativistic contribution to the total relativistic eigenenergy) is also determined in analytical form. To our knowledge, any analytical form of relativistic effect for any potential has never been reported.

In the following section, a step-by-step method of deriving the solutions of the Schrödinger equation from the KleinGordon equation is proposed. A brief review on the bound state solutions for Morse potential follows. The detailed arithmetic procedure, which describes i) how to set up the Klein-Gordon equation for the Morse potential, ii) how to exactly solve it to have the exact relativistic bound state solutions, and iii) how to derive the nonrelativistic solutions from the exact relativistic solutions, is presented. Conclusions and discussion are provided in the last section.

\section{From Relativistic Solutions to Nonrelativistic Solutions}

The goal is to obtain the bound state solutions of nonrelativistic Schrödinger equation (Eq. 2) with potential $W(x)$ from the exact bound state solutions of relativistic Klein-Gordon equation (Eq. 1) with potentials, $V(x)$ and $S(x)$. The approach in Ref. 25 is extended and a more elaborate step-by-step method is suggested as follows.

Step 1. Set up a Schrödinger equation for potential $W(x)$ whose nonrelativistic solutions are desired. Because bound states are of interest, proper boundary conditions must be pre-determined.

Step 2. Properly choose a scalar potential, $S(x)$ and a vector potential, $V(x)$ and set up a relevant Klein-Gordon equation. The requirements for the choice are: (i) The sum of $S(x)$ and $V(x)$ should be equal to $W(x)$. (ii) The two potential functions, $S(x)$ and $V(x)$ must have a form for which the Klein-Gordon equation can be exactly solved for bound states with the same boundary conditions of the Schrödinger equation with $W(x)$. (iii) In the nonrelativistic limit, the potential energies $(|S|$ and $|V|)$ should be very small compared to the rest mass energy, $m c^{2}$.

The choice of two potentials is crucial so that a care must be taken. The following findings may be helpful in choosing the two potentials: When the scalar potential energy $(|S|)$ is larger than the vector potential energy $(|V|)$, the Klein-Gordon equation always has analytical bound state solutions. Sometimes though the relativistic Klein-Gordon equation produces bound states, the corresponding nonrelativistic Schrödinger equation may not have bound states for the same potentials or vice versa. For example, when $S(x)=-V(x)$, the Klein-Gordon equation can have bound states but the Schrödinger equation has continuum states.

Step 3. Solve the relativistic Klein-Gordon equation to have the exact relativistic bound state solutions. See the requirement (ii) in Step 2. In case one can solve the Klein-Gordon equation exactly, not only the nonrelativistic solutions but also the relativistic effect can be obtained. When one wants the nonrelativistic solutions only, the asymptotic solutions of the KleinGordon equation are sufficient enough for the purpose. The relativistic Klein-Gordon equation produces two distinct solutions, one for particle states and the other for antiparticle states.
The particle state solutions should be used in the next step because the nonrelativistic Schrödinger equation is defined for particles only.

Step 4. Derive the nonrelativistic solutions in the nonrelativistic limit where the relativistic effect is negligibly small,. The derivation can be achieved in two ways. One is to take a limit expression of the relativistic solutions when a parameter in potentials $(S(x), V(x))$ is very small and then subtract the rest mass energy, $m c^{2}$. The other is to apply the approximation $E \cong m c^{2}$ that is valid in the nonrelativistic limit to the relativistic solutions and then subtract the rest mass energy, $m c^{2}$. See the requirement (iii) in Step 2.

\section{Nonrelativistic Bound State Solutions for Morse Potential}

The Morse potential energy function is frequently used to describe the vibrational motion (Morse oscillator) of diatomic molecules or the electronic motion of electrons in small atoms. The one-dimensional Schrödinger equation for the Morse potential can be exactly solved for bound states, and the analytical form of solutions (eigenenergies and eigenfunctions) are well known. $^{26-29}$

The nonrelativistic Schrödinger equation for the Morse potential is

$$
\begin{aligned}
-\frac{\hbar^{2}}{2 m} \frac{d^{2}}{d x^{2}} \Psi^{\text {nonrel }}(x) & +D_{e}[1-\exp (-\beta x)]^{2} \Psi^{\text {nonrel }}(x) \\
& =E^{\text {nonrel }} \Psi^{\text {nonrel }}(x)(-\infty<x<\infty) .
\end{aligned}
$$

$D_{e}$ and $\beta$ are adjustable positive numbers (or parameters). The Morse potential has a minimum value of zero at $x=0$. It is infinite at $x=-\infty$ and is $D_{e}$ at $x=\infty$. Comparing with Eq. (2), the potential is $W(x)=D_{e}[1-\exp (-\beta x)]^{2}$.

The bound state eigenenergies $E^{\text {nonrel }}$ with boundary conditions of $\Psi^{\text {nonrel }}(-\infty)=\Psi^{\text {nonrel }}(\infty)=0$ are

$$
\begin{aligned}
E^{\text {nonrel }}=D_{e}-\left[\sqrt{D_{e}}-\frac{\beta \hbar}{\sqrt{2 m}}\left(n+\frac{1}{2}\right)\right]^{2} & \\
& \left(n=0,1,2, \ldots, n_{\max }\right)
\end{aligned}
$$

where $n_{\max }$ is the largest integer less than $\frac{\sqrt{2 m D_{e}}}{\beta \hbar}$. Note that the eigenenergies are all positive. The smallest eigenenergy (when $n=0$ ) is $\beta \hbar \sqrt{\frac{D_{e}}{2 m}}-\frac{\beta^{2} \hbar^{2}}{8 m}$ and the largest eigenenergy (when $n=$ $n_{\text {max }}$ ) is less than $D_{e}$.

The orthonormalized bound state eigenfunctions $\Psi^{\text {nonrel }}(x)$ are, in terms of new variables $z=2 \lambda \exp (-\beta x)$ and $\lambda=\frac{\sqrt{2 m D_{e}}}{\beta \hbar}$,

$$
\Psi^{\text {nonrel }}(z)=N z^{\lambda-n-\frac{1}{2}} \exp \left(-\frac{1}{2} z\right) L_{n}^{2 \lambda-2 n-1}(z)
$$

where the normalization constant is $N=[\Gamma(n+1) \Gamma(2 \lambda-n)]^{-1 / 2}$. $L_{n}^{2 \lambda-2 n-1}(z)$ is a Laguerre polynomial and $\Gamma(n+1)$ is a gamma 
function.

In the following section, the method suggested in the previous section is applied to the Morse potential and the step-by-step procedure for determining the nonrelativistic solutions from the relativistic Klein-Gordon equation is presented.

\section{From Relativistic to Nonrelativistic Solutions for Morse Potential}

Step 1. The nonrelativistic Schrödinger equation of interest is given in Eq. (3). The nonrelativistic solutions to derive are eigenenergies $E^{\text {nonrel }}\left(\right.$ Eq. 4) and eigenfunctions $\Psi^{\text {nonrel }}(x)$ (Eq. 5). The potential is $W(x)=S(x)+V(x)=D_{e}[1-\exp (-\beta x)]^{2}$. The relevant boundary conditions are $\Psi^{\text {nonrel }}(-\infty)=\Psi^{\text {nonrel }}(\infty)=0$.

Step 2. Let $S(x)=a[1-\exp (-\beta x)]^{2}$ and $V(x)=b[1-\exp$ $(-\beta x)]^{2}$ where $a+b=D_{e}$, then the relativistic Klein-Gordon equation is

$$
\begin{gathered}
-\hbar^{2} c^{2} \frac{d^{2}}{d x^{2}} \Psi(x)+\left(m c^{2}+a[1-\exp (-\beta x)]^{2}\right)^{2} \Psi(x) \\
=\left(E-b[1-\exp (-\beta x)]^{2}\right)^{2} \Psi(x)
\end{gathered}
$$

with boundary conditions of $\Psi(-\infty)=\Psi(\infty)=0$. This choice of two potentials obviously satisfies the requirement (i) explained in the former section.

The requirement (ii) dictates that $S(x)$ and $V(x)$ must have a form for which the Klein-Gordon equation can be exactly solved. Suppose we choose $S(x) \neq V(x)$ (i.e., $a \neq b)$, then the Klein-Gordon equation (Eq. 6) can be rewritten as

$$
\begin{aligned}
-\frac{\hbar^{2}}{2 m} & \frac{d^{2}}{d x^{2}} \Psi(x)+\left[\frac{a^{2}-b^{2}}{2 m c^{2}} D_{e}^{2}[1-\exp (-\beta x)]^{4}\right. \\
& \left.+\left(\frac{m c^{2} a+E b}{m c^{2}}\right) D_{e}[1-\exp (-\beta x)]^{2}\right] \Psi(x)=\varepsilon \Psi(x)
\end{aligned}
$$

with $\varepsilon=\frac{E^{2}-m^{2} c^{4}}{2 m c^{2}}$. This Klein-Gordon equation (Eq. 7) cannot be exactly solved because of the $[1-\exp (-\beta x)]^{4}$ term present. Furthermore, it does not have bound state solutions when $b^{2}>a^{2}$. As mentioned before, it verifies that the Klein-Gordon equation does not necessarily have bound state solutions when the vector potential energy $(|V|)$ is larger than the scalar potential energy $(|S|)$. Therefore, for the Morse potential, the choice of $S(x) \neq V(x)$ violates the requirement (ii). In consequence, $S(x)$ must be equal to $V(x)$, i.e., $a=b=D_{e} / 2$ and the KleinGordon equation to be solved is

$$
\begin{array}{r}
-\hbar^{2} c^{2} \frac{d^{2}}{d x^{2}} \Psi(x)+\left(m c^{2}+\frac{1}{2} D_{e}[1-\exp (-\beta x)]^{2}\right)^{2} \Psi(x) \\
=\left(E-\frac{1}{2} D_{e}[1-\exp (-\beta x)]^{2}\right)^{2} \Psi(x) \quad(-\infty<x<\infty)
\end{array}
$$

The requirement (iii) is automatically satisfied in the nonrelativistic limit, because the potential parameter $D_{e}$ for a particle, under a usual chemical environment, is very small compared to the term, $m c^{2}$.

Step 3. The Klein-Gordon equation (Eq. 8) can be rewritten as

$$
\begin{aligned}
& -\frac{\hbar^{2}}{2 m} \frac{d^{2}}{d x^{2}} \Psi(x)+D_{e}\left(\frac{m c^{2}+E}{2 m c^{2}}\right)[1-\exp (-\beta x)]^{2} \Psi(x) \\
& \quad=\varepsilon \Psi(x)
\end{aligned}
$$

with $\varepsilon \equiv \frac{E^{2}-m^{2} c^{4}}{2 m c^{2}}$. Eq. (9) formally mimics the Schrödinger equation. It is known that the Klein-Gordon equation can always be reduced to a Schrödinger-type equation when $S(x)=V(x) .^{21}$ It is a textbook knowledge to solve the above Klein-Gordon equation that has a form of Schrödinger equation for Morse potential, but here one should solve it iteratively and consistently because the energy $E$ appears in the potential function.

Let the initial guess for $E$ be $E^{(0)}$. Solving Eq. (9) with boundary conditions of $\Psi(-\infty)=\Psi(\infty)=0$, one obtains the energylike term $\varepsilon$,

$$
\begin{aligned}
& \varepsilon=\frac{E^{2}-m^{2} c^{4}}{2 m c^{2}}=\frac{D_{e}\left(m c^{2}+E^{(0)}\right)}{2 m c^{2}} \\
&-\left[\sqrt{\frac{D_{e}\left(m c^{2}+E^{(0)}\right)}{2 m c^{2}}}-\frac{\beta \hbar}{\sqrt{2 m}}\left(n+\frac{1}{2}\right)\right]^{2}
\end{aligned}
$$

where the quantum number $n$ (the number of nodes in $\Psi(x)$ ) is $0,1,2, \ldots$

Solving Eq. (10) for $E$, one obtains the first iterative energy $E^{(1)}$,

$$
\begin{gathered}
E^{(1)}= \pm \sqrt{m^{2} c^{4}+2 m c^{2}\left\{D_{e}\left(\frac{1}{2}+\frac{E^{(0)}}{2 m c^{2}}\right)\right.} \\
\left.-\left[D_{e}^{1 / 2}\left(\frac{1}{2}+\frac{E^{(0)}}{2 m c^{2}}\right)^{1 / 2}-\frac{\beta \hbar}{\sqrt{2 m}}\left(n+\frac{1}{2}\right)\right]^{2}\right\} \\
= \pm \sqrt{m^{2} c^{4}+2 m c^{2}\left[K^{(1)}-\left(K^{(1)^{1 / 2}}-\frac{\beta \hbar}{\sqrt{2 m}}\left(n+\frac{1}{2}\right)\right)^{2}\right]}
\end{gathered}
$$

where

$$
K^{(1)}=D_{e}\left(\frac{1}{2}+\frac{E^{(0)}}{2 m c^{2}}\right) \text {. }
$$

Since only particle states have meanings in this work, the + sign must be chosen, i.e., 


$$
E^{(1)}=m c^{2} \sqrt{1+\frac{2}{m c^{2}}\left[K^{(1)}-\left(K^{(1)^{1 / 2}}-\frac{\beta \hbar}{\sqrt{2 m}}\left(n+\frac{1}{2}\right)\right)^{2}\right]} .
$$

The first iterative normalized eigenfunction $\Psi^{(1)}(z)$ is

$$
\begin{aligned}
\Psi^{(1)}(z)= & {\left[\Gamma(n+1) \Gamma\left(2 \lambda^{(1)}-n\right)\right]^{-1 / 2} } \\
& z^{\lambda^{(1)}-n-\frac{1}{2}} \exp \left(-\frac{1}{2} z\right) L_{n}^{2 \lambda^{(1)}-2 n-1}(z)
\end{aligned}
$$

with $z=2 \lambda^{(1)} \exp (-\beta x)$ and $\lambda^{(1)}=\frac{\sqrt{2 m K^{(1)}}}{\beta \hbar}$.

Inserting $E^{(1)}$ into $E$ in Eq. (9) and solving it again, one obtains the second iterative energy $E^{(2)}$,

$$
E^{(2)}=m c^{2} \sqrt{1+\frac{2}{m c^{2}}\left[K^{(2)}-\left(K^{(2)^{1 / 2}}-\frac{\beta \hbar}{\sqrt{2 m}}\left(n+\frac{1}{2}\right)\right)^{2}\right]}
$$

where

$$
\begin{aligned}
K^{(2)}= & D_{e}\left(\frac{1}{2}+\frac{E^{(1)}}{2 m c^{2}}\right)=D_{e}\left\{\frac{1}{2}+\frac{1}{2 m c^{2}}\left(m c^{2} \sqrt{1+\frac{2}{m c^{2}}}\right.\right. \\
& {\left.\left.\left[K^{(1)}-\left(K^{(1)^{1 / 2}}-\frac{\beta \hbar}{\sqrt{2 m}}\left(n+\frac{1}{2}\right)\right)^{2}\right]\right)\right\} } \\
= & D_{e}\left\{\frac{1}{2}+\frac{1}{2 m c^{2}}\left(m c^{2} \sqrt{1+\frac{2}{m c^{2}}\left[D_{e}\left(\frac{1}{2}+\frac{E^{(0)}}{2 m c^{2}}\right)\right.}\right.\right. \\
& \left.\left.\left.-\left(D_{e}^{1 / 2}\left(\frac{1}{2}+\frac{E^{(0)}}{2 m c^{2}}\right)^{1 / 2}-\frac{\beta \hbar}{\sqrt{2 m}}\left(n+\frac{1}{2}\right)\right)^{2}\right]\right)\right\}
\end{aligned}
$$

The second iterative eigenfunction $\Psi^{(2)}(z)$ is

$$
\begin{aligned}
\Psi^{(2)}(z)= & {\left[\Gamma(n+1) \Gamma\left(2 \lambda^{(2)}-n\right)\right]^{-1 / 2} } \\
& z^{\lambda^{(2)}-n-\frac{1}{2}} \exp \left(-\frac{1}{2} z\right) L_{n}^{2 \lambda^{(2)}-2 n-1}(z)
\end{aligned}
$$

with $z=2 \lambda^{(2)} \exp (-\beta x)$ and $\lambda^{(2)}=\sqrt{2 m K^{(2)}} / \beta \hbar$.

After infinite number of iterations, one obtains the $E^{(\infty)}$ that must be equal to the exact energy $E$, i.e.,

$$
\begin{aligned}
E & =E^{(\infty)} \\
& =m c^{2} \sqrt{1+\frac{2}{m c^{2}}\left[K^{(\infty)}-\left(K^{(\infty)^{1 / 2}}-\frac{\beta \hbar}{\sqrt{2 m}}\left(n+\frac{1}{2}\right)\right)^{2}\right]}
\end{aligned}
$$

$$
\begin{aligned}
K^{(\infty)}= & D_{e}\left(\frac{1}{2}+\frac{E^{(\infty)}}{2 m c^{2}}\right) \\
= & D_{e}\left\{\frac{1}{2}+\frac{1}{2 m c^{2}}\left(m c^{2} \sqrt{1+\frac{2}{m c^{2}}\left[D_{e}\left(\frac{1}{2}+\frac{1}{2 m c^{2}}(\cdots)\right)\right.}\right.\right. \\
& \left.\left.\left.-\left(D_{e}^{1 / 2}\left(\frac{1}{2}+\frac{1}{2 m c^{2}}(\cdots)\right)^{1 / 2}-\frac{\beta \hbar}{\sqrt{2 m}}\left(n+\frac{1}{2}\right)\right)^{2}\right]\right)\right\}
\end{aligned}
$$

Here $(\cdots)$ denotes the infinite repetition of the term, labeled as $y$,

$$
\begin{aligned}
& y \equiv m c^{2} \sqrt{1+\frac{2}{m c^{2}}\left[D_{e}\left(\frac{1}{2}+\frac{1}{2 m c^{2}}(\cdots)\right)\right.} \\
& \left.-\left(D_{e}^{1 / 2}\left(\frac{1}{2}+\frac{1}{2 m c^{2}}(\cdots)\right)^{1 / 2}-\frac{\beta \hbar}{\sqrt{2 m}}\left(n+\frac{1}{2}\right)\right)^{2}\right] .
\end{aligned}
$$

Note that the value of $y$ becomes independent of the choice of $E^{(0)}$ because there is the infinite number of terms embedded.

Then one immediately finds that, from Eqs. (18) and (20), the exact relativistic energy $E$ is

$$
E=E^{(\infty)}=y
$$

and, inserting Eq. (20) into Eq. (18), one also obtains another expression for $E$, i.e.,

$$
E=m c^{2} \sqrt{1+\frac{2}{m c^{2}}\left[D_{e}\left(\frac{1}{2}+\frac{1}{2 m c^{2}} y\right)\right.}
$$

$$
\left.-\left(D_{e}^{1 / 2}\left(\frac{1}{2}+\frac{1}{2 m c^{2}} y\right)^{1 / 2}-\frac{\beta \hbar}{\sqrt{2 m}}\left(n+\frac{1}{2}\right)\right)^{2}\right] .
$$

Equating Eq. (21) with Eq. (22), one obtains

$$
\begin{aligned}
& E=m c^{2} \sqrt{1+\frac{2}{m c^{2}}\left[D_{e}\left(\frac{1}{2}+\frac{1}{2 m c^{2}} E\right)\right.} \\
& \overline{\left.-\left(D_{e}^{1 / 2}\left(\frac{1}{2}+\frac{1}{2 m c^{2}} E\right)^{1 / 2}-\frac{\beta \hbar}{\sqrt{2 m}}\left(n+\frac{1}{2}\right)\right)^{2}\right] .}
\end{aligned}
$$

Rearranging Eq. (23), one obtains the quartic equation for $E$,

where 


$$
\begin{aligned}
E^{4} & -2 c^{2}\left\{\beta^{2} \hbar^{2}(n+1 / 2)^{2}-m^{2} c^{2}\right\} E^{2} \\
& -4 c^{2} \beta^{2} \hbar^{2}(n+1 / 2)^{2} D_{e} E \\
& +c^{4}\left\{\beta^{2} \hbar^{2}(n+1 / 2)^{2}-m^{2} c^{2}\right\}^{2} \\
& -4 m c^{4} \beta^{2} \hbar^{2}(n+1 / 2)^{2} D_{e}=0
\end{aligned}
$$

Therefore one can obtain the exact eigenenergies $E$ for the relativistic Klein-Gordon equation (Eq. 8) by solving the above quartic equation.

As many authors suggested, ${ }^{4-16}$ there is a simple and direct way of solving Eq. (9) without iteration. Let us assume that the exact $E$ is already known, i.e., $E$ is a constant. Then Eq. (9) can be regarded as a Schrödinger equation with Morse potential and its bound state energy $\varepsilon$ is

$$
\begin{aligned}
\varepsilon= & \frac{E^{2}-m^{2} c^{4}}{2 m c^{2}}=\frac{D_{e}\left(m c^{2}+E\right)}{2 m c^{2}} \\
& -\left[\sqrt{\frac{D_{e}\left(m c^{2}+E\right)}{2 m c^{2}}}-\frac{\beta \hbar}{\sqrt{2 m}}\left(n+\frac{1}{2}\right)\right]^{2} .
\end{aligned}
$$

Rearranging it, one obtains

$$
\begin{aligned}
& E=m c^{2} \sqrt{1+\frac{2}{m c^{2}}\left[\frac{D_{e}\left(m c^{2}+E\right)}{2 m c^{2}}\right.} \\
& \left.-\left(\sqrt{\frac{D_{e}\left(m c^{2}+E\right)}{2 m c^{2}}}-\frac{\beta \hbar}{\sqrt{2 m}}\left(n+\frac{1}{2}\right)\right)^{2}\right] .
\end{aligned}
$$

The Eq. (26) is identical with Eq. (23) obtained from the iterative procedure. Therefore this direct method is found to be adequate. However, in general, the direct method is valid only when the Klein-Gordon equation is exactly solvable.

The exact relativistic eigenfunction $\Psi(z)\left(=\Psi^{(\infty)}(z)\right)$ is

$$
\begin{aligned}
\Psi(z)= & {\left[\Gamma(n+1) \Gamma\left(2 \lambda^{(\infty)}-n\right)\right]^{-1 / 2} } \\
& z^{\lambda^{(\infty)}-n-\frac{1}{2}} \exp \left(-\frac{1}{2} z\right) L_{n}^{2 \lambda^{(\infty)}-2 n-1}(z)
\end{aligned}
$$

with $z=2 \lambda^{(\infty)} \exp (-\beta x)$ and $\lambda^{(\infty)}=\sqrt{2 m K^{(\infty)}} / \beta \hbar$.

Step 4. The direct way of obtaining $E^{\text {nonrel }}$ is to solve the quartic equation (Eq. 24) to have the relativistic $E$ and take a limit value of $E$ when $D_{e}<<1$. But there is a simple alternate way for determining $E^{\text {nonrel }}$ by using Eq. (23) instead of Eq. (24). When the potential energies ( $|S|$ and $|V|)$ are small compared to $m c^{2}$, one immediately notices that the approximation $E \cong m c^{2}$ is valid in the nonrelativistic limit.

Imposing the approximation $E \cong m c^{2}$ on Eq. (23), one obtains

$$
E \cong m c^{2} \sqrt{1+\frac{2}{m c^{2}}\left[D_{e}-\left(D_{e}^{1 / 2}-\frac{\beta \hbar}{\sqrt{2 m}}\left(n+\frac{1}{2}\right)\right)^{2}\right]} .
$$

When $D_{e}<<1$, the second argument in the square root is much smaller than 1 so that the square root can be approximated as

$$
\begin{aligned}
E \cong m c^{2}\{1 & +\left(\frac{1}{2}\right) \frac{2}{m c^{2}}\left[D_{e}-\left(D_{e}^{1 / 2}\right.\right. \\
& \left.\left.\left.-\frac{\beta \hbar}{\sqrt{2 m}}\left(n+\frac{1}{2}\right)\right)^{2}\right]+\mathcal{O}\left(\frac{D_{e}^{2}}{m^{2} c^{4}}\right)\right\}
\end{aligned}
$$

where $\mathcal{O}\left(\frac{D_{e}^{2}}{m^{2} c^{4}}\right)$ is the higher order term (or contribution).

Ignoring the higher order term, the relativistic eigenenergy is

$$
E \cong m c^{2}+\left[D_{e}-\left(D_{e}^{1 / 2}-\frac{\beta \hbar}{\sqrt{2 m}}\left(n+\frac{1}{2}\right)\right)^{2}\right]
$$

Removing the rest mass energy from Eq. (30), finally one obtains the nonrelativistic energy $E^{\text {nonrel }}$ in the nonrelativistic limit, i.e.,

$$
E^{\text {nonrel }} \cong D_{e}-\left(D_{e}^{1 / 2}-\frac{\beta \hbar}{\sqrt{2 m}}\left(n+\frac{1}{2}\right)\right)^{2}
$$

which is identical with Eq. (4).

In the nonrelativistic limit where the approximation $E \cong m c^{2}$ is valid, from Eq. (19), one obtains $K^{(\infty)} \cong D_{e}$. Therefore the nonrelativistic eigenfunction is, from Eq. (27),

$$
\begin{aligned}
\Psi^{\text {nonrel }}(z)= & {[\Gamma(n+1) \Gamma(2 \lambda-n)]^{-1 / 2} } \\
& z^{\lambda-n-\frac{1}{2}} \exp \left(-\frac{1}{2} z\right) L_{n}^{2 \lambda-2 n-1}(z)
\end{aligned}
$$

with $z=2 \lambda \exp (-\beta x)$ and $\lambda=\sqrt{2 m D_{e}} / \beta \hbar$.

Note that Eq. (32) is identical with the true nonrelativistic eigenfunction, Eq. (5).

We have successfully derived the eigenenergies and eigenfunctions of the nonrelativistic Schrödinger equation from the relativistic Klein-Gordon equation. As seen in Eqs. (31) and (32), we have obtained the exact nonrelativistic solutions. It is due to the fact that, for the Morse potential, the relativistic KleinGordon equation can be exactly transformed to the nonrelativistic Schrödinger equation in the nonrelativistic limit. ${ }^{30}$

The higher order term in Eq. (29) representing the relativistic effect which is an amount of relativistic contribution to eigenenergy. In the nonrelativistic limit, i.e., for a particle whose kinetic 
energy is small and the potentials acting on the particle are not large (this is a situation frequently encountered in chemistry), is

$$
\begin{aligned}
E(\text { relativistic effect })=\mathcal{O}\left(\frac{D_{e}^{2}}{m^{2} c^{4}}\right) & -\frac{1}{8}\left\{\frac{2}{m c^{2}}\left[D_{e}-\left(D_{e}^{1 / 2}-\frac{\beta \hbar}{\sqrt{2 m}}\left(n+\frac{1}{2}\right)\right)^{2}\right]\right\}^{2} \\
& +\frac{1}{16}\left\{\frac{2}{m c^{2}}\left[D_{e}-\left(D_{e}^{1 / 2}-\frac{\beta \hbar}{\sqrt{2 m}}\left(n+\frac{1}{2}\right)\right)^{2}\right]\right\}^{3}+\cdots \\
\cong & \left.-\frac{1}{2 m^{2} c^{4}}\left[D_{e}-\left(D_{e}^{1 / 2}-\frac{\beta \hbar}{\sqrt{2 m}}\left(n+\frac{1}{2}\right)\right)^{2}\right]^{2}\right]^{2} \\
\cong & -\frac{\left(E^{\text {nonrel }}\right)^{2}}{2 m^{2} c^{4}} .
\end{aligned}
$$

As seen in Eq. (33), the relativistic effect is negative which is usual for a bound particle. ${ }^{31}$ It is usually positive for an antiparticle. Since $0<E^{\text {nonrel }}<D_{e}$, the magnitude of relativistic effect ranges from almost zero to $D_{e}^{2} / 2 m^{2} c^{4}$. As expected, in the usual situation the relativistic effect is very small because $D_{e}<<m c^{2}$. For the higher states ( $n$ is large) the relativistic effect in magnitude becomes larger because the particle moves faster at the higher states.

\section{Conclusions and Discussion}

A detailed step-by-step method for analytically deriving the bound state solutions of nonrelativistic Schrödinger equation from relativistic Klein-Gordon equation is suggested. In the current work, the iterative approach of solving the Klein-Gordon is adopted so that one is able to derive the asymptotic solutions of the corresponding Schrödinger equation with ease. The feasibility of the suggested method is tested by applying it to the Morse potential. Furthermore, this new method enables us to derive the relativistic effect for a slow moving particle under the Morse potential. To our knowledge it is the first work to separate out the relativistic effect from the total eigenenergy in an analytical manner.

Despite the feats that this method has achieved, there are still many details that should be more studied. Firstly, the current method is an ad doc procedure. To set up a Klein-Gordon equation one has to choose appropriate scalar and vector potentials. Though there are some constraints in choosing $S(x)$ and $V(x)$ (see the requirements (i), (ii), and (ii) in Step 2), mathematically there are infinite possibilities of choosing each of the scalar and vector potential functions. Therefore more systematic approach is necessary. Secondly, there are few potential functions whose exact relativistic solutions can be analytically obtained by solving the Klein-Gordon equation. It makes this method have limited applications. Probably, for inexactly solvable potentials, an effort to directly search for asymptotic solutions is more desirable than the current method. A research toward this direction is worthwhile to pursue in the future. Thirdly, the derivation procedure (Step 4) is not systematic in mathematical sense. In the nonrelativistic limit, the approximation $E \cong m c^{2}$ is surely valid. However there is no guarantee that it is the sole approximation that describes the correct nonrelativistic limit. Last, the example of the Morse potential is not sufficient enough to definitely confirm the usefulness of this method because the Schrödinger equation for the Morse potential can be exactly solved. A more convincing example, such as a potential whose relativistic solutions are solvable but nonrelativistic solutions are not solvable, should be presented. Further studies along this direction are under way.

At the first glance, this work seems to be counter-intuitive because the solutions of the relatively simple Schrödinger equation are derived from the more complicated Klein-Gordon equation. It is a sort of induction approach frequently exploited in experiments. But we have shown that this induction approach could be useful even in theories.

\section{References}

1. Klein, O. Z. Phys. 1926, 37, 895.

2. Gordon, W. Z. Phys. 1927, 40, 117.

3. Greiner, W. Relativistic Quantum Mechanics; Springer: Berlin, 1990.

4. Jana, T. K.; Roy P. Phys. Lett. A 2009, 373, 1239.

5. Jana, T.; Roy, P. Phys. Lett. A 2007, 361, 55.

6. Chen, G.; Chen, Z.; Xuan, P. Phys. Lett. A 2006, 352, 317.

7. Diao, Y.; Yi, L.; Jia, C. Phys. Lett. A 2004, 332, 157.

8. Chen, G. Phys. Lett. A 2005, 339, 300.

9. de Castro, A. S. Phys. Lett. A 2005, 338, 81.

10. de Souza Dutra, A.; Chen, G. Phys. Lett. A 2006, 349, 297.

11. Qiang, W.-C.; Zhou, R.-S.; Gao, Y. Phys. Lett. A 2007, 371, 201.

12. Chen, G.; Chen, Z.-D.; Lou, Z.-M. Phys. Lett. A 2004, 331, 374.

13. Mehmet, S.; Harun, E. J. Phys. A: Math. Gen. 2004, 37, 4379.

14. Chen, G. Mod. Phys. Lett. A 2004, 19, 2009.

15. Yi, L.; Diao, Y.; Liu, J.; Jia, C. Phys. Lett. A 2004, 333, 212.

16. Garcia, M. G.; de Castro, A. S. Ann. Phys. 2009, 324, 2372.

17. Znojil, M. J. Phys. A: Math. Gen. 2004, 37, 9557.

18. Barakat, T. Ann. Phys. 2009, 324, 725.

19. McQuarrie, B. R.; Vrscay, E. R. Phys. Rev. A 1993, 47, 868.

20. Barton, G. J. Phys. A: Math. Theor. 2007, 40, 1011.

21. Hall, R. L. Phys. Lett. A 2007, 372, 12.

22. Alhaidari, A. D.; Bahlouli, H.; Al-Hasan, A. Phys. Lett. A 2006, $349,87$.

23. Zhao, X.-Q.; Jia, C.-S.; Yang, Q.-B. Phys. Lett. A 2005, 337, 189.

24. Hall, R. L.; Lucha, W. Phys. Lett. A 2010, 374, 1980.

25. Sun, H. Phys. Lett. A 2009, 374, 116.

26. Morse, P. M. Phys. Rev. 1929, 34, 57.

27. Herzberg, G. Molecular Spectra and Molecular Structure I. Spectra of Diatomic Molecules; Van Nostrand Reinhold: New York, 1950.

28. Cooper, F.; Khare, A.; Sukhatme, U. Phys. Rep. 1995, 251, 267.

29. Sun, H. Phys. Lett. A 2005, 338, 309.

30. Sun, H. to be published.

31. Pilkuhn, H. M. Relativistic Quantum Mechanics; Springer: New York, 2003. 